Del Giudice, E. and A. De Ninno. Are Nuclear Transmutations Observed At Low Energies Consequences Of Qed Coherence? in Tenth International Conference on Cold Fusion. 2003. Cambridge, MA: LENR-CANR.org. This paper was presented at the 10th International Conference on Cold Fusion. It may be different from the version published by World Scientific, Inc (2003) in the official Proceedings of the conference.

\title{
Are Nuclear Transmutations Observed At Low Energies Consequences Of Qed Coherence?
}

\author{
E. DEL GIUDICE \\ INFN Milano - Via Celoria 16, 20133 Milano (Italy) \\ E_mail:del giudice@infn.mi.it \\ A. DE NINNO, A. FRATTOLILLO \\ ENEA Frascati - Via Enrico Fermi 27, 00044 Roma (Italy)
}

\begin{abstract}
Nuclear transmutations have been reported to occur in matrices subjected to either electrochemical or gas loading at room temperature. To overcome the difficulties of the large Coulomb repulsion among nuclei a $\gamma$-ray electromagnetic field appears as a suitable agent. It is discussed whether this e.m. excitation could emerge from cold fusion processes and induce nuclear reactions through the giant resonance coupling of this e.m. field with the closed shells present in the nuclei of the matrix.
\end{abstract}

\section{Low Energy Nuclear Transmutations}

Many reports [1], [2], [3] point to the existence of nuclear transmutations occurring in solid metal matrices when they are loaded with hydrogen isotopes beyond a threshold. Elements absent before the loading were found and the natural abundancies of the isotopes of the host metal were modified.

These results look "unreasonable" in the framework of conventional nuclear physics. Quite large energies (several MeV's) are needed to get the same reactions starting from reactant nuclei forming an ensemble of independent particles.

These energies cannot be produced in any conceptual frame where phonon excitations only are present. A major conceptual difficulty arises from the large Coulomb barrier among the nuclei, whose overcoming would require large amounts of energy. Actually only the fusion of nuclei (deuterons) having $\mathrm{Z}=1$ could be made possible by the mechanisms of enhancement produced by coherence [4], since the dependence upon $Z$ of the Gamov penetration factor is exponential the probability of the barrier crossing for $Z>1$ is negligible. The only possible agents for nuclear transmutations should be in our case the uncharged ones: neutrons or e.m. fields. However the existence of sizeable sources of neutrons within the lattice should be excluded, so that the only surviving agent is the e.m. field.

It has been pointed out $[4,5]$ that during processes of cold fusion very strong e.m. fields having the frequency of $\gamma$ rays appear for very short intervals of time. Let us briefly summarize the main points of this dynamic.

\section{Coherent Dynamics in Cold Fusion}

a)In a Pd crystal at room temperature the d-electron shells are in a coherent regime within "coherence domain" (CD) as large as some hundreds of Angstroms. Electron shells oscillate in tune with a coherent e.m. field trapped in the $\mathrm{CD}$, whose frequency is in the range of soft $\mathrm{X}$-rays $(v<100 \mathrm{eV})$. The coherent plasma of the d-shells is so stiff to produce at selected 
points in the lattice permanent blobs of negative charge able to catalyze nuclear fusion in a way akin to the muon catalyzed fusion. This catalysis amounts to increase the barrier penetration factor among deuterons by about 40 orders of magnitude

b)Hydrogens filling the metal enter into a coherent state when $x>0.6$. The corresponding CD's range between 1 and $10 \mu \mathrm{m}$ and oscillations are tuned with a self trapped e.m. field, whose frequency is in the IR interval.

c)In the case of deuterium, when $x>1$, the above coherent state induces a further magnification of the probability of tunneling of deuterons across the Coulomb barrier making possible the large number of fusions needed to produce the observed large amounts of excess heat (see G. Preparata reports at several ICCF's)

The excess energy is also released in a time shorter than that required to split the "boiling" ${ }^{4} \mathrm{He}$ nucleus by the nuclear dynamics (about $10^{-21} \mathrm{sec}$.), so preventing a massive emission of neutron and tritium [5].

\section{Energy output of C.F.}

Let us analyze in more details the process of release of energy in C.F.

The production of $4 \mathrm{He}$ unaccompanied by $\gamma$-rays demands a very fast $\left(\mathrm{t}<10^{-21} \mathrm{sec}\right)$ energy transfer to the lattice electrons. Suppose that a fusion be a physical event localized at a definite site in the lattice. In order to reach the nearest atom at a distance of about $1 \AA$ the velocity $\mathrm{v}$ of the energy transfer should be about $300 \mathrm{c}$ !

According to universally accepted principles of physics it is impossible to transfer the energy of a fusion dd $\rightarrow{ }^{4} \mathrm{He}$ (without $\gamma$ rays) to the lattice.

So cold fusion cannot be a localized event !

There are examples in quantum physics of non-localized events (e.g. the Mössbauer effect) occurring within extended coherent regions such as the CD's of deuterons filling the Pd lattice. In a CD each participant is delocalized, being spread out on the whole volume of the CD.

As in the Mössbauer effect, each event implying a participant is delocalized on the whole $\mathrm{CD}$, so that energy doesn't need to travel in order to be transferred to other physical subjects present in the same volume.

The coherent plasma of deuterons is so robust that the large energy release of the nuclear fusion can be shared among macroscopic numbers of elementary systems [5]

Let us call $\Phi \mathrm{Dp}$ the wave field of the deuteron plasma and $\eta_{\mathrm{D}}$ the wave function of the additional deuteron $\mathrm{D}$ involved in the reaction

$$
\mathrm{D}+\mathrm{D} \mathrm{p}->\text { nuclear state NS + lattice energy }
$$

Let's call $|N S(t)\rangle$ the time dependent wave function of the nuclear state,

$A$ the coherent e.m. vector potential of the $\mathrm{CD}, J$ the e.m. current, $\mathrm{HN}_{\mathrm{N}}$ the nuclear Hamiltonian

$$
i \frac{\partial}{\partial t}|N S(t)\rangle=\left(H_{N}+e A_{k} J_{k}\right)|N S(t)\rangle
$$

By applying perturbation theory, we get the transition amplitude of the energy transfer from fusing nuclei to the field over the time $\mathrm{T}$

$$
A(D D p \rightarrow N S ; T)=-i \int_{0}^{T} d t\langle N S| H \text { int }|D D p\rangle
$$




$$
H \text { int }=e \int d^{3} x \vec{A}(\vec{x}, t) \cdot \vec{J}(\vec{x}, t)
$$

from which the average transition rate over the time $\mathrm{T}$ is

$$
R(D D p \rightarrow N S ; T)=\frac{|A(D D p \rightarrow N S ; T)|^{2}}{T}
$$

Following the mathematical treatment of Chapt. 8 of the quoted book, one gets the energy per CD released over the time

$$
T=\frac{2 \pi}{\varpi_{r}} \approx 10^{-14}
$$

$$
\varpi_{C D}=(x-1) N_{C D} R(D D p \rightarrow N S) \delta E \approx 10^{-8} \quad \text { W/CD }
$$

where NCD is the number of components in the $\mathrm{CD},(\mathrm{x}-1)$ is assumed to be 0.1 and $\delta \mathrm{E} \sim 24$ $\mathrm{MeV}$ is the energy released by a single fusion.

Considering that a $\mathrm{cm}^{3}$ contains $1012 \mathrm{CD}$ 's one gets the power per $\mathrm{cm}^{3}$

$$
\mathrm{W}=10 \mathrm{KW} / \mathrm{cm}^{3}
$$

in good agreement with observations.

The previous treatment allows us also to evaluate the time $\mathrm{T}_{0}$ needed for releasing the energy of $3.4 \mathrm{MeV}$ necessary to put the "boiling" nucleus below the threshold of splitting. One gets

$$
\mathrm{T} 0 \sim 1 / 210^{-21} \mathrm{sec}<10^{-21} \mathrm{sec}
$$

that is the time required by nuclear dynamics to split the nucleus (see the report of Preparata at the Conference in Salt Lake City in 1990).

In conclusion the output energy of each fusion, which appears at first as an excitation of the deuteron CD e.m. field having a $\gamma$-ray frequency, is transferred speedily to the Pd electron plasma, where appears as an excitation at much lower frequencies.

There is however, a very short time interval where a short lived e.m. field having the frequency of $\gamma$-rays is present in the CD.

At the rate $(10 \mathrm{KW} / \mathrm{cc})$ estimated above, one can calculate that in each $\mathrm{CD}$ there is in the average 1 fusion each $4 * 10^{-4}$ seconds. The energy released by each fusion is transferred to 
the lattice in a time of $10^{-14}$ seconds, so that there is no superposition between the two events.

Each fusion produces a $\gamma$-ray e.m. field lasting about $10^{-21} \mathrm{sec}$ and spread out on the whole volume of the $\mathrm{CD}$.

\section{Giant dipole resonance (GDR) induced by $\gamma$-ray e.m. fields}

Almost sixty years ago Arkadii Beinusovich Migdal [6], Baldwin and Klaiber [7]] and Maurice Goldhaber and Edward Teller [8] discovered the phenomenon of GDR, which is a wide maximum of the interaction between $\gamma$-photons and nuclei located at $14-15 \mathrm{MeV}$, e.g. fig.1.

GDR is connected with the excitation of quantum collective modes in nuclei. The peak energy, as shown by Goldhaber \& Teller, coincides with the binding energy of two closed shells in a nucleus. In a sense, under the $\gamma$-field, the nucleus enters a vibration able to break the binding, so releasing single nucleons or full shells.

It is conceivable that the extended $\gamma$-ray field has a very high probability to interact with at least one nucleus enclosed in the CD and induce a GDR.

Actually the frequency of the $\gamma$-ray field is, for at least $10^{-21} \mathrm{sec}$, in the range between 16 and $24 \mathrm{MeV}$

\section{Conclusions}

This contribution is still a seminal one.

A detailed calculation of the interaction parameters of the coupling of the extended $\gamma$-ray field (which is not a single photon!) with nuclei is needed.

However the existence of such extended $\gamma$-ray fields is a necessary consequence of the coherent theory of cold fusion. Such fields appear, in the frame of accepted principles of quantum physics, as the likeliest engine to give rise to nuclear transmutations in the metal lattice at room temperature. 


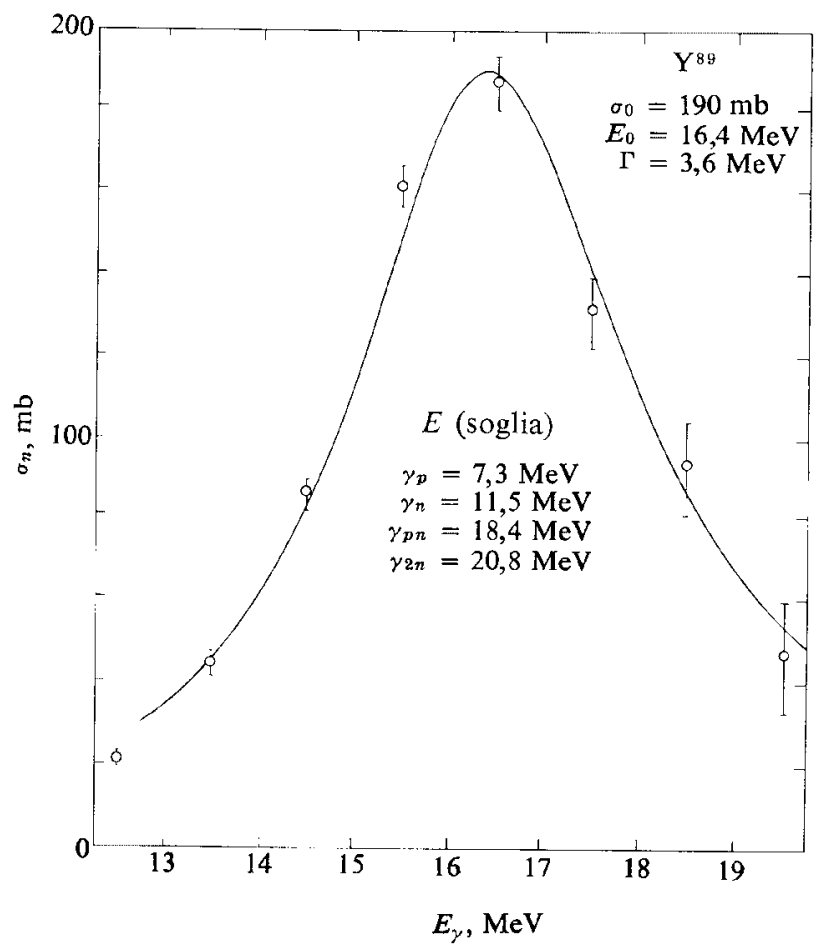

Fig.1 Neutron photoproduction cross-section in ${ }^{89}$ Y. [Yergin and Fabricand, Phys.Rev., 104,1334,1956]

\section{References}

[1] Y. Iwamura, in Proc. of ICCF9 (2002) , p.141

[2] T. Passel, in Proc. of ICCF9 (2002) , p.299

[3] T. Ohmori, in Proc. of ICCF9 (2002) , p.284

[4] T. Bressani, E. Del Giudice, G. Preparata, Nuovo Cim. 101A, (1989), 845

[5] G. Preparata "QED in Condensed Matter" World Scientific, 1995

[6] A.B. Migdal Zh. Eksp. Theor. Fiz. 15 (1945) 81

[7] Baldwin and Klaiber Phys.Rev. 71 (1947) 3

[8] M. Goldhaber, E. Teller Phys. Rev. 74 (1948) 1046 